Editor's Note: Toolboxes are intended to briefly highlight a new method or a resource of general use in neuroscience or to critically analyze existing approaches or methods. For more information, see http://www.jneurosci.org/misc/itoa.shtml.

\title{
The Serial Reaction Time Task: Implicit Motor Skill Learning?
}

\author{
Edwin M. Robertson \\ Center for Non-Invasive Brain Stimulation, Beth Israel Deaconess Medical Center, Boston, Massachusetts 02215
}

Since its development 20 years ago, the serial reaction time task (SRTT) has gone from being a tool used by psychologists (Nissen and Bullemer, 1987) to one that, in the last few years, has been embraced by a wider community (Fig. 1). Embedded within this task is a sequence, a connected series of events, that engages processes supporting the temporal organization of behavior, the formation of high-order associations, and the prediction of future events (Keele et al., 2004; Chafee and Ashe, 2007). Thus, this task has been used to explore the processes underlying a broad range of behaviors, including the cognitive and biological principles of learning and memory. Despite this success, it remains a task that is frequently misunderstood. Questions surround how task performance should be measured, whether it is appropriate to conceptualize the SRTT as an implicit learning task, whether it is a motor task, and whether it is dependent on the integrity of the medial temporal lobe (MTL). Here we briefly describe the SRTT and discuss these questions. An appreciation of these questions will aid the design and interpretation of future neuroscientific experiments.

\footnotetext{
Received June 16, 2007; revised Aug. 7, 2007; accepted Aug. 7, 2007.

This work was supported by National Institutes of Health Grant R01 NS051446. I am grateful to Alvaro Pascual-Leone and Matt Walker for their thoughtful and constructive comments.

Correspondence should be addressed to Edwin M. Robertson, Center for Non-Invasive Brain Stimulation, Beth Israel Deaconess Medical Center, 330 Brookline Avenue, Kirstein Building KS 454, Boston, MA 02215. E-mail: emrobert@bidmc.harvard.edu.

D0I:10.1523/JNEUROSCI.2747-07.2007

Copyright $\odot 2007$ Society for Neuroscience $\quad 0270-6474 / 07 / 2710073-03 \$ 15.00 / 0$
}

The serial reaction time task

In this task, a visual cue can appear at any one of four positions arranged horizontally on a computer screen. Each screen position, designated $1-4$, corresponds to a button on a response pad. When a cue appears, at the start of each trial, a participant selects the appropriate response button, which ends the trial (Fig. 1). The duration of each trial, defined by the participant's response time, is the primary task measure. At the end of each trial, there is a short fixed delay, often between 200 and $500 \mathrm{~ms}$, before another cue is presented. The visual cues play out a repeating sequence of positions (for example, 2-3-1-4-3-2-4-1-3-4-2-1). These sequential trials are then followed by random trials in which the visual cue no longer plays out a repeating pattern of positions. Thus, the SRTT is an apparently straightforward four-choice reaction time task containing a repeating sequence that participants come to predict, and so learn. However, there are a number of misunderstandings and open questions that continue to surround this seductively simple task. Here we briefly touch on some of these issues.

\section{Measuring skill learning}

In the SRTT, a simple measure of skill may be to measure the gradual reduction in response time that takes place across the sequential trials (Fig. 1). This provides a measure of participants' growing expertise in performing not only the sequence but also in learning the visuomotor association, or mapping, between the position of the visual cue and the required response. This latter component should not be underestimated: when a visuomotor association is learned in isolation, as participants perform random trials, there can be substantial reductions in response time (Fig. 1). Thus, a change in sequential response time, although simple, supplies a general measure of participants' competence at performing the SRTT. A more specific measure of skill learning can be obtained by contrasting the sequential response times against those response times for the random trials. Not only does this analysis provide a specific measure of skill, it also minimizes the possible contaminating influences of factors such as fatigue and motivation. There is also a subtle but nonetheless important advantage of using the difference between sequential and random response times over using sequential response times alone. When the sequence is unexpectedly removed and replaced with random trials, the participant initially continues to inappropriately play out the sequence. This mistake inflates the random response times, increasing the difference between the sequential and random response times. This implies that the difference measure of skill is not only specific but is also highly sensitive to participants' expectation that the visual cues will play out a sequence of positions. Thus, the difference between sequential and random response times provides a specific and sensitive measure of skill acquisition in the SRTT. 


\section{Implicit learning}

Skill learning, and particularly that acquired with the SRTT, is often portrayed as an example of implicit learning. Although an individual can be unaware of learning (implicit learning), it is also possible for an individual to be aware of learning a new skill (explicit learning). A challenging problem has been to define when individuals have become aware of learning. One of the strengths of the SRTT is that there are many distinct and complementary techniques available for measuring awareness. For example, implicit learning can be defined behaviorally as recalling only a few items of the sequence during a free recall test or having a poor recognition for segments of the sequence during a recognition test (Curran, 1997; Willingham and Goedert-Eschmann, 1999). Alternatively, others have sought to define implicit learning neuropsychologically as the skill that can be acquired by amnesiacs who can neither recall nor recognize any aspect of the learned sequence (Reber and Squire, 1994). These measures assume that awareness and declarative knowledge for the sequence, although distinct, are developing in parallel. Thus, tests of declarative knowledge are viewed as tests of awareness. Such an assumption may be justified. However, others have suggested that all tests of implicit learning are flawed because they lack the necessary sensitivity or specificity to detect awareness (Hannula et al., 2005). This perspective, however, assumes that there is a single moment at which an individual is transformed from being unaware to being aware of learning and that this moment can be measured, whereas awareness may be a continuum. Thus, any attempt to define a particular moment of awareness could be arbitrary. Innovative techniques may address this problem by allowing the continuum of an individual's awareness to be mapped out (Persaud et al., 2007). Unfortunately, as yet, there is no consensus about how to measure an individual's awareness for learning in this task. Nonetheless, some component of learning is likely achieved implicitly, and there is an array of complementary techniques that can provide insight into individuals' awareness for learning.

An elegant technique that overcomes many of the problems associated with measuring awareness is to directly manipulate individuals' awareness for the sequence. This can be achieved by instructing individuals that a particular color of visual stimuli is associated with a sequence, while participants remain un-

(A)
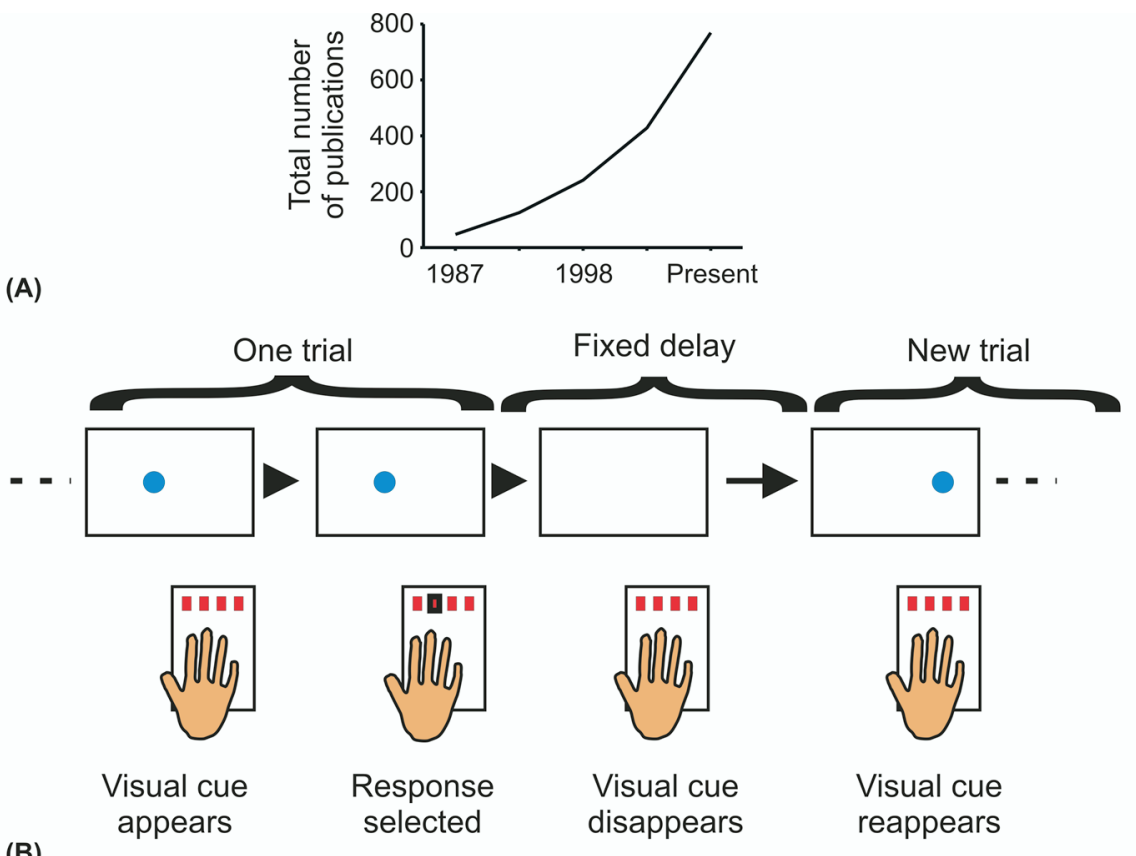

(B)

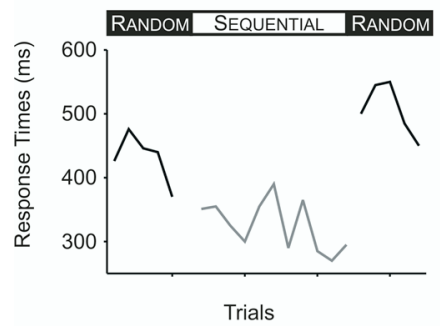

(C)

Figure 1. A, Using the SRTT has become increasingly popular: as many studies using the SRTT have taken place in the last 5 years as were conducted in the preceding 15 years. $\boldsymbol{B}$, A schematic of the SRTT: a visual cue appears, a participant responds by selecting the appropriate response button, the visual cue disappears, ending the trial, and after a fixed delay, another visual cue appears marking the beginning of a new trial. The position of the visual cue can either play out a repeating sequence or be random. C, Response time data from a single participant, showing a steady decrease over the initial random and sequential trials until the reintroduction of the random trials, when there is a dramatic increase in response time. The magnitude of this increase is often used as a measure of sequence learning in the SRTT (Willingham et al., 1989). D. The MTL is activated during SRTT sequence learning, and a circuit that includes the MTL makes a critical contribution to this learning (Curran, 1997; Schendan et al., 2003).

aware that another color of visual stimuli is also playing out a sequence (Willingham et al., 2002; Robertson et al., 2004). Thus an individual is aware of learning one sequence but unaware of learning the other sequence. By contrasting between these conditions of explicit and implicit sequence learning, it is possible to gain insight into the effects of awareness on brain activation and behavior. Thus, without supplying a definitive solution to the challenging problem of measuring awareness, it is possible to gain insight into the cognitive and biological features of awareness.

\section{Motor learning}

Although the SRTT is often viewed as a motor learning task, it is not clear that learning is taking place solely within the motor domain. The serial regularity could be learned as a sequence of motor responses with participants learning the correct sequence of response buttons. Alternatively, the serial regularity could be learned perceptually as a sequence of visual cue positions: participants would learn to predict the position of each visual cue and so would respond quickly to the appearance of the visual cue. Recent work has shown that sequence learning can be supported by purely perceptual learning; however, this occurs when the SRTT has been modified to remove the motor sequence (Dennis et al., 2006). With no motor sequence, individuals are forced to use the only regularity available, present within the perceptual domain, and so learn the sequence perceptually. In contrast, when both a perceptual and a motor 
sequence are available, learning is no longer purely perceptual (Willingham, 1999). Thus, the SRTT is not simply a motor learning task; it can have both motor and perceptual learning components. Recognizing that perceptual learning is a component of the SRTT is important. It provides an explanation for the shift in brain areas supporting SRTT performance when the perceptual properties of the SRTT are altered (Robertson et al., 2001).

When performing the SRTT, individuals acquire skill at producing a sequence, plus they can also acquire an ability to declaratively describe the sequence. Having performed the SRTT, an individual might be able to verbally describe some or all of the sequence contained within the task. This combination of skill plus declarative knowledge for that skill is present within many everyday behaviors. For example, we can skillfully produce a sequence of finger movements to tap out a phone number, plus we are also able to tell others the number to call. Thus the SRTT is not exclusively a motor learning task; it includes an important declarative component, demonstrated by our ability to verbally describe some or all of the sequence within the SRTT. Recognizing that declarative learning is also a component of the SRTT provides an explanation for how learning a word list can influence task performance: word-list learning disrupts the declarative component and so allows the off-line enhancement of the procedural component of the SRTT (Brown and Robertson, 2007).

The medial temporal lobe and the SRTT Typically, motor skill learning is supported by a circuit that includes motor cortical areas and subcortical areas such as the striatum and the cerebellum. A similar pattern of areas are engaged during learning in the SRTT (Keele et al., 2004). The prefrontal cortex, striatum, and cerebellum all make critical contributions to SRTT performance improvements (Pascual-Leone et al., 1993; Robertson et al., 2001; Torriero et al., 2004). Insight into the nature of these contributions is possible through subtle but simple changes to the configuration of the SRTT. This has made it possible to tease apart the neural circuits responsible for supporting learning within different coordinate frames or to examine the influence of awareness on the engagement of brain areas (Willingham et al., 2002; Cohen et al., 2005). Thus, the SRTT can act as a bridge between brain and behavior, providing insights into the computational contribution that brain areas make to sequence learning. A poignant example of this capacity is provided by the differential engagement of the MTL during SRTT performance. The MTL is engaged and makes a critical contribution to the acquisition of high- but not low-order sequences even when participants are not declaratively learning any of the sequence (Curran, 1997; Schendan et al., 2003). Predicting the next event (i.e., $n$ ) within a high-order sequence requires knowledge of the two immediately preceding events [i.e., $(n-$ 2 ) plus $(n-1)]$, whereas predicting loworder sequences only requires knowledge of the preceding event [i.e., $(n-1)]$. The MTL is engaged to support learning when there is a greater temporal component to the SRTT. Similarly, the MTL does not make a critical contribution to classical conditioning until this simple form of motor learning is modified to include a temporal component, as occurs in trace conditioning (Solomon et al., 1986). Thus, engagement of the MTL is related not to the type of behavior being supported (e.g., declarative vs procedural), but instead to the computational requirements of that task. This principle may be applicable to the engagement of other brain areas.

\section{Conclusion}

The SRTT is a seductively simple task, appearing to be just a four-choice reaction time task that provides a convenient measure of implicit motor skill learning. Here we have described several of the misconceptions that surround this task to reveal a powerfully sophisticated task that can provide insights into a wider and richer array of human behaviors than its popular classification would imply. Appreciating the subtle complexity of this task will provide an aid to the design and interpretation of experiments.

\section{References}

Brown RM, Robertson EM (2007) Inducing motor skill improvements with a declarative task. Nat Neurosci 10:148-149.

Chafee MV, Ashe J (2007) Intelligence in action. Nat Neurosci 10:142-143.

Cohen DA, Pascual-Leone A, Press DZ, Robertson EM (2005) Off-line learning of motor skill memory: a double dissociation of goal and movement. Proc Natl Acad Sci USA 102:18237-18241.

Curran T (1997) Higher order associative learning in amnesia: evidence from the serial reaction time task. J Cogn Neurosci 9:522-533.

Dennis NA, Howard Jr JH, Howard DV (2006) Implicit sequence learning without motor sequencing in young and old adults. Exp Brain Res 175:153-164.

Hannula DE, Simons DJ, Cohen NJ (2005) Imaging implicit perception: promise and pitfalls. Nat Rev Neurosci 6:247-255.

Keele S, Ivry RB, Mayr U, Hazeltine E, Heuer H (2004) The cognitive and neural architecture of sequence representation. Psychol Rev 110:316-339.

Nissen MJ, Bullemer P (1987) Attentional requirements of learning: evidence from performance measures. Cognit Psychol 19:1-32.

Pascual-Leone A, Grafman J, Clark K, Stewart M, Massaquoi S, Lou JS, Hallett M (1993) Procedural learning in Parkinson's disease and cerebellar degeneration. Ann Neurol 34:594-602.

Persaud N, McLeod P, Cowey A (2007) Postdecision wagering objectively measures awareness. Nat Neurosci 10:257-261.

Reber PJ, Squire LR (1994) Parallel brain systems for learning with and without awareness. Learn Mem 1:217-229.

Robertson EM, Tormos JM, Maeda F, PascualLeone A (2001) The role of the dorsolateral prefrontal cortex during sequence learning is specific for spatial information. Cereb Cortex 11:628-635.

Robertson EM, Pascual-Leone A, Press DZ (2004) Awareness modifies the skill-learning benefits of sleep. Curr Biol 14:208-212.

Schendan HE, Searl MM, Melrose RJ, Stern CE (2003) An FMRI study of the role of the medial temporal lobe in implicit and explicit sequence learning. Neuron 37:1013-1025.

Solomon PR, Vander Schaaf ER, Thompson RF, Weisz DJ (1986) Hippocampus and trace conditioning of the rabbit's classically conditioned nictitating membrane response. Behav Neurosci 100:729-744.

Torriero S, Oliveri M, Koch G, Caltagirone C, Petrosini L (2004) Interference of left and right cerebellar rTMS with procedural learning. J Cogn Neurosci 16:1605-1611.

Willingham DB (1999) Implicit motor sequence learning is not purely perceptual. Mem Cognit 27:561-572.

Willingham DB, Goedert-Eschmann K (1999) The relation between implicit and explicit learning: evidence for parallel development. Psychol Sci 10:531-534.

Willingham DB, Nissen MJ, Bullemer P (1989) On the development of procedural knowledge. J Exp Psychol Learn Mem Cogn 15:1047-1060.

Willingham DB, Salidis J, Gabrieli JD (2002) Direct comparison of neural systems mediating conscious and unconscious skill learning. J Neurophysiol 88:1451-1460. 